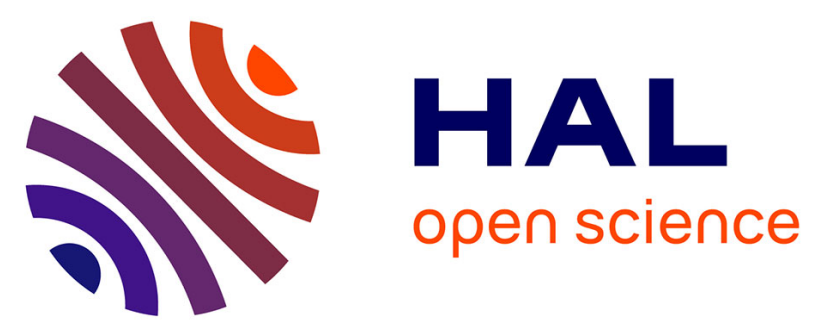

\title{
Diffusion in a nonhomogeneous medium: quasi-random walk on a lattice
}

Rami El Haddad, Christian Lécot, Gopalakrishnan Venkiteswaran

\section{To cite this version:}

Rami El Haddad, Christian Lécot, Gopalakrishnan Venkiteswaran. Diffusion in a nonhomogeneous medium: quasi-random walk on a lattice. Monte Carlo Methods and Applications, 2010, 16, pp.211230. hal-00949605

\section{HAL Id: hal-00949605 https://hal.univ-grenoble-alpes.fr/hal-00949605}

Submitted on 19 Feb 2014

HAL is a multi-disciplinary open access archive for the deposit and dissemination of scientific research documents, whether they are published or not. The documents may come from teaching and research institutions in France or abroad, or from public or private research centers.
L'archive ouverte pluridisciplinaire HAL, est destinée au dépôt et à la diffusion de documents scientifiques de niveau recherche, publiés ou non, émanant des établissements d'enseignement et de recherche français ou étrangers, des laboratoires publics ou privés. 


\title{
Diffusion in a nonhomogeneous medium: quasi-random walk on a lattice
}

\author{
Rami El Haddad, Christian Lécot and Gopalakrishnan Venkiteswaran
}

\begin{abstract}
We are interested in Monte Carlo (MC) methods for solving the diffusion equation: in the case of a constant diffusion coefficient, the solution is approximated by using particles and in every time step, a constant stepsize is added to or substracted from the coordinates of each particle with equal probability. For a spatially dependent diffusion coefficient, the naive extension of the previous method using a spatially variable stepsize introduces a systematic error: particles migrate in the directions of decreasing diffusivity. A correction of stepsizes and stepping probabilities has recently been proposed and the numerical tests have given satisfactory results. In this paper, we describe a quasi-Monte Carlo (QMC) method for solving the diffusion equation in a spatially nonhomogeneous medium: we replace the random samples in the corrected MC scheme by low-discrepancy point sets. In order to make a proper use of the better uniformity of these point sets, the particles are reordered according to their successive coordinates at each time step. We illustrate the method with numerical examples: in dimensions 1 and 2, we show that the QMC approach leads to improved accuracy when compared with the original $\mathrm{MC}$ method using the same number of particles.
\end{abstract}

Keywords. Quasi-Monte Carlo, random walk, low-discrepancy sequences, diffusion equation.

AMS classification. $11 \mathrm{~K} 45,80 \mathrm{M} 25$.

\section{Introduction}

Random walk techniques are useful computational tools for simulating processes involving the diffusion of substances. The substance is modelled by a set of particles and the particles move according to the dynamics described in the diffusion equation. For diffusion in one dimension and in the case of a constant diffusion coefficient $D_{0}$, there are two main ways of performing random walk. A short time interval $\Delta t$ is chosen. With the first method, a particle with coordinate $x$ moves to $x+\Delta x$, where $\Delta x$ is a random increment drawn from a centered Gaussian distribution with variance $2 D_{0} \Delta t$. This approach is discussed in $[1,8,7]$. The second method consists of using a constant stepsize $\Delta x:=\sqrt{2 D_{0} \Delta t}$. A particle with coordinate $x$ jumps to $x-\Delta x$ or to $x+\Delta x$ with equal probability $1 / 2$. This is the approach taken in $[11,6,9]$. Both methods are simple to implement and allow to consider complicated geometries. Drawbacks are their slow convergence so that many runs are often needed to obtain reliable results. 
When considering a problem with a spatially dependent diffusion coefficient, i.e. $D=D(x)$, the naive extensions of the above methods are to use a spatially variable variance $2 D(x) \Delta t$ in the first case, and a spatially variable stepsize $\sqrt{2 D(x) \Delta t}$ in the second case. However, both approaches give biased results as they lead to an apparent advection in directions of decreasing diffusivity and a concentration of particles in regions of low diffusivity $[10,4,5]$. A systematic method for calculating corrections to the Gaussian steplength has been proposed by L. Farnell and W. G. Gibson in [4]. The same authors have given in [5] a method for correcting the fixed step case: both the steplength and the probability of stepping to left and right must be modified. Numerical experiments have shown that both corrected schemes lead to satisfactory results.

One approach to improve the accuracy of MC methods is to change the random numbers used. QMC methods replace pseudo-random numbers with quasi-random numbers. These are points which are very evenly distributed. We refer to [17] for detailed information on QMC methods. In MC integration, it is not the randomness of the samples that is relevant, but rather that they are spread in a uniform manner on the integration domain, so QMC quadrature often outperforms MC integration . But in particle simulations the independence of the random samples is important. If care is not taken in how the quasi-random points are used, the correlations may destroy the method. An additionnal technique was first introduced in [12] and consists of sorting the particles according to position at each time step. This technique was applied to the diffusion equation (in spatially homogeneous media) in $[16,2,14,18]$. In a previous communication [3], we have proposed and tested a QMC algorithm for the simulation of the diffusion equation based on the corrected Gaussian steplength of [4]. The aim of the present work is to develop a QMC version of the corrected MC algorithm of [5].

The rest of the paper is organized as follows. Section 2 presents the classical random walk scheme with constant stepsize and its deterministic version, for the simulation of diffusion in an one-dimensional homogeneous medium. In Section 3, we recall the corrections of the steplength and probability in order to deal with spatially variable diffusion coefficients. Section 4 presents a QMC version of the corrected MC scheme proposed above. In Section 5, we illustrate the new method with numerical examples in dimensions one and two, and we show that the accuracy is improved by the QMC approach, when compared with the standard MC strategy. We draw conclusions in the final section.

\section{Random walk on a lattice}

The diffusion equation describes the evolution of the density of a substance due to the displacement of molecules (attributed to Brownian motion) from regions of strong concentration to regions of small concentration. The pure initial value problem for a 
simple diffusion equation in a one-dimensional domain is:

$$
\begin{aligned}
\frac{\partial c}{\partial t}(x, t) & =\frac{\partial}{\partial x}\left(D \frac{\partial c}{\partial x}\right)(x, t), \quad x \in \mathbb{R}, t>0 \\
c(x, 0) & =c_{0}(x), \quad x \in \mathbb{R},
\end{aligned}
$$

where $D:=D(x)>0$ is the diffusion coefficient and $c_{0}(x) \geq 0$ is the initial concentration at position $x$. Here $c(x, t)$ is the concentration of particles at location $x$ and time $t$. If we assume

$$
\int_{\mathbb{R}} c_{0}(x) d x=1
$$

then

$$
\forall t>0 \quad \int_{\mathbb{R}} c(x, t) d x=1 .
$$

In the spatially homogeneous case, $D(x):=D_{0}$, the random walk technique on a lattice can be described as follows. Let $\Delta x$ be a spatial step and $\Delta t$ be a time step. The finite difference method replaces Eq. (2.1) with

$$
\frac{c(x, t+\Delta t)-c(x, t)}{\Delta t} \approx \frac{D_{0}}{\Delta x^{2}}(c(x-\Delta x, t)-2 c(x, t)+c(x+\Delta x, t)) .
$$

Hence, if $\Delta x=\sqrt{2 D_{0} \Delta t}$, one has

$$
c(x, t+\Delta t) \approx \frac{1}{2} c(x-\Delta x, t)+\frac{1}{2} c(x+\Delta x, t) .
$$

Given a large integer $N$, the simulation is conducted by first generating $N$ samples (particles) from the initial probability density function $c_{0}$. Then the particles random walk as follows. During a small time step $\Delta t$, each particle moves by a random distance

$$
L_{0}:=\sqrt{2 D_{0} \Delta t} B
$$

where $B$ is a Bernoulli random variable such that $\mathbf{P}(B=-1)=\mathbf{P}(B=+1)=1 / 2$.

A QMC version of the previous scheme has been analyzed in [2]. The basic idea of QMC methods is to replace the pseudo-random points of MC methods by lowdiscrepancy point sets. We first recall from [17] some basic concepts. Denote $I:=$ $[0,1)$ and let $s$ be a fixed dimension and $\lambda_{s}$ be the $s$-dimensional Lebesgue measure. For a set $U=\left\{\mathbf{u}_{0}, \ldots, \mathbf{u}_{N-1}\right\}$ of points in the $s$-dimensional unit cube $I^{s}$, we define the discrepancy by

$$
D_{N}(U):=\sup _{J}\left|\frac{1}{N} \sum_{0 \leq k<N} 1_{J}\left(\mathbf{u}_{k}\right)-\lambda_{s}(J)\right|,
$$

where $1_{J}$ denotes the indicator function of $J$, and where the supremum is taken over all subintervals $J$ of $I^{s}$. We say that a set of $N$ points of $I^{s}$ is a low-discrepancy 
point set if its discrepancy is of order $\mathcal{O}\left((\log N)^{s-1} / N\right)$, which is the minimum size possible. The theory of $(t, m, s)$-nets and $(t, s)$-sequences was developed with a view to providing excellent low-discrepancy point sets for QMC methods. For an integer $b \geq 2$, an elementary interval in base $b$ is an interval of the form

$$
\prod_{i=1}^{s}\left[a_{i} b^{-d_{i}},\left(a_{i}+1\right) b^{-d_{i}}\right)
$$

with integers $d_{i} \geq 0$ and $0 \leq a_{i}<b^{d_{i}}$ for $1 \leq i \leq s$. If $0 \leq t \leq m$ are integers, a $(t, m, s)$-net in base $b$ is a point set consisting of $b^{m}$ points in $I^{s}$ such that every elementary interval $J$ in base $b$ of measure $b^{t-m}$ contains exactly $b^{t}$ points of the set. If $b \geq 2$ and $t \geq 0$ are integers, a sequence $\left\{\mathbf{u}_{0}, \mathbf{u}_{1}, \ldots\right\}$ of points in $I^{s}$ is called a $(t, s)$-sequence in base $b$ if, for all integers $n \geq 0$ and $m>t$, the points $\mathbf{u}_{j}$ with $n b^{m} \leq j<(n+1) b^{m}$ form a $(t, m, s)$-net in base $b$.

We sketch the quasi-random walk method for the simulation of (2.1)-(2.2) in the case of a constant diffusion coefficient $D_{0}$. The first step towards the approximation of the solution entails the discretization of the space variable $x$ only. Let $\Delta x$ be a space step and $\tau_{ \pm \Delta x}$ be the operator defined by

$$
\tau_{ \pm \Delta x} f(x):=f(x \pm \Delta x)
$$

for any function $f$ on $\mathbb{R}$. The semi-discrete approximation to (2.1)-(2.2) is

$$
\begin{aligned}
\frac{\partial \widetilde{c}}{\partial t}(x, t) & =\frac{D_{0}}{\Delta x^{2}}\left(\tau_{-\Delta x} \widetilde{c}-2 \widetilde{c}+\tau_{\Delta x} \widetilde{c}\right)(x, t), \quad x \in \mathbb{R}, t>0, \\
\widetilde{c}(x, 0) & =c_{0}(x), \quad x \in \mathbb{R} .
\end{aligned}
$$

It is easy to show that the conservation law still holds:

$$
\forall t>0 \quad \int_{\mathbb{R}} \widetilde{c}(x, t) d x=1 .
$$

We first construct a weak formulation of the equation. A mapping $\sigma: \mathbb{R} \rightarrow \mathbb{R}$ is said to be simple if $\sigma(\mathbb{R})$ is a finite set. Let $\mathcal{S}_{+}(\mathbb{R})$ be the set of all nonnegative simple measurable functions on $\mathbb{R}$. Then, for any $\sigma \in \mathcal{S}_{+}(\mathbb{R})$,

$$
\frac{d}{d t} \int_{\mathbb{R}} \sigma(x) \widetilde{c}(x, t) d x=\frac{D_{0}}{\Delta x^{2}} \int_{\mathbb{R}}\left(\tau_{-\Delta x} \sigma-2 \sigma+\tau_{\Delta x} \sigma\right)(x) \widetilde{c}(x, t) d x .
$$

Let $b$ and $m$ be integers and denote $N:=b^{m}$. We generate $N$ samples (particles) $x_{k}^{0}, 0 \leq k<N$ from the initial probabillity distribution $c_{0}(x) d x$, i.e.

$$
c^{0}(x):=\frac{1}{N} \sum_{0 \leq k<N} \delta\left(x-x_{k}^{0}\right) \approx c_{0}(x) d x
$$


where $\delta\left(x-x^{*}\right)$ denotes the Dirac measure at $x^{*}$. Next we discretize time into intervals of length $\Delta t$ with the following stability requirement

$$
\lambda:=D_{0} \frac{\Delta t}{\Delta x^{2}} \leq \frac{1}{2}
$$

For QMC integration, we need a low discrepancy sequence: $U=\left\{\mathbf{u}_{0}, \mathbf{u}_{1}, \ldots\right\} \subset I^{2}$. For an integer $n$, denote $U^{n}:=\left\{\mathbf{u}_{j}: n N \leq j<(n+1) N\right\}$. If $\Pi_{1}$ is the map defined by $\Pi_{1}\left(u_{1}, u_{2}\right):=u_{1}$, we assume that for all $n \in \mathbb{N}$,

$$
\Pi_{1} U^{n} \text { is a }(0, m, 1) \text {-net in base } b .
$$

Assuming that we have computed an approximation $c^{n}$ of $\widetilde{c}$ at time $t_{n}:=n \Delta t$ by a sum of Dirac masses located at positions $x_{0}^{n}, \ldots, x_{N-1}^{n}$ :

$$
c^{n}(x):=\frac{1}{N} \sum_{0 \leq k<N} \delta\left(x-x_{k}^{n}\right),
$$

we compute an approximation $c^{n+1}$ at time $t_{n+1}$ in two steps.

(1) Sorting the particles. The particles are relabeled such that

$$
x_{0}^{n} \leq x_{1}^{n} \leq \cdots \leq x_{N-1}^{n} .
$$

This reordering was first introduced in [12] for the simulation of the Boltzmann equation. It guarantees the convergence of the method: since a step of the random walk can be described by a numerical integration (see below), the sorting reverts to minimizing the amplitude of the jumps of the function to be integrated.

(2) Quasi-random walk. A measure $\gamma^{n+1}(x)$ which approximates the solution at time $t_{n+1}$ is obtained by using a forward Euler scheme to discretize Eq. (2.11): for any $\sigma \in \mathcal{S}_{+}(\mathbb{R})$,

$$
\frac{1}{\Delta t} \int_{\mathbb{R}} \sigma(x)\left(\gamma^{n+1}(x)-c^{n}(x)\right)=\frac{D_{0}}{\Delta x^{2}} \int_{\mathbb{R}}\left(\tau_{-\Delta x} \sigma-2 \sigma+\tau_{\Delta x} \sigma\right)(x) c^{n}(x) .
$$

Consequently,

$$
\int_{\mathbb{R}} \sigma(x) \gamma^{n+1}(x)=\frac{1}{N} \sum_{0 \leq k<N}\left(\lambda \sigma\left(x_{k}^{n}-\Delta x\right)+(1-2 \lambda) \sigma\left(x_{k}^{n}\right)+\lambda \sigma\left(x_{k}^{n}+\Delta x\right)\right) .
$$

Denote by $1_{k}$ the indicator function of the elementary interval $I_{k}:=[k / N,(k+1) / N)$ and let $\chi_{-1}, \chi_{0}$ and $\chi_{+1}$ denote, respectively, the indicator functions of the intervals $I_{-}:=[0, \lambda), I_{0}:=[\lambda, 1-\lambda), I_{+}:=[1-\lambda, 1)$. To $\sigma \in \mathcal{S}_{+}(\mathbb{R})$, we associate

$$
\Sigma^{n}(\mathbf{u}):=\sum_{0 \leq k<N} 1_{k}\left(u_{1}\right) \sum_{\varepsilon=-1,0,+1} \chi_{\varepsilon}\left(u_{2}\right) \sigma\left(x_{k}^{n}+\varepsilon \Delta x\right), \quad \mathbf{u}=\left(u_{1}, u_{2}\right) \in I^{2} .
$$


Then

$$
\int_{\mathbb{R}} \sigma(x) \gamma^{n+1}(x)=\int_{I^{2}} \Sigma^{n}(\mathbf{u}) d \mathbf{u} .
$$

We recover $c^{n+1}$ by performing a QMC integration:

$$
\int_{\mathbb{R}} \sigma(x) c^{n+1}(x)=\frac{1}{N} \sum_{n N \leq j<(n+1) N} \Sigma^{(n)}\left(\mathbf{u}_{j}\right) .
$$

This can be reworded as follows. For $u \in[0,1)$, put $k(u):=\lfloor N u\rfloor$, where $\lfloor\xi\rfloor$ denotes the greatest integer $\leq \xi$. It follows from (2.13) that the mapping

$$
j \in\{n N, n N+1, \ldots,(n+1) N-1\} \mapsto k\left(u_{j, 1}\right) \in\{0,1, \ldots, N-1\}
$$

is a bijection. Then, the random walk restatement of (2.18) reads: for $n N \leq j<$ $(n+1) N$,

$$
x_{k\left(u_{j, 1}\right)}^{n+1}= \begin{cases}x_{k\left(u_{j, 1}\right)}^{n}-\Delta x, & \text { if } 0 \leq u_{j, 2}<\lambda \\ x_{k\left(u_{j, 1}\right)}^{n}, & \text { if } \lambda \leq u_{j, 2}<1-\lambda \\ x_{k\left(u_{j, 1}\right)}^{n}+\Delta x, & \text { if } 1-\lambda \leq u_{j, 2}<1 .\end{cases}
$$

If $\lambda=1 / 2$, this reduces to:

$$
x_{k\left(u_{j, 1}\right)}^{n+1}= \begin{cases}x_{k\left(u_{j, 1}\right)}^{n}-\Delta x, & \text { if } 0 \leq u_{j, 2}<1 / 2, \\ x_{k\left(u_{j, 1}\right)}^{n}+\Delta x, & \text { otherwise }\end{cases}
$$

which must be compared with (2.7). For every $j$, the first coordinate $u_{j, 1}$ of the quasirandom point $\mathbf{u}_{j}$ is used to select the particle, while the second coordinate $u_{j, 2}$ is used to decide whether the particle moves left or right. This approach may be extended to several dimensions. In [2], convergence of the algorithm is established in the multidimensional case; the results of computational experiments indicate that a significant improvement is achieved over standard random walk simulation.

In some applications, the diffusion coefficient $D(x)$ varies with position. In this case, the obvious extension of the previous method using a spatially variable space step $\sqrt{2 D(x) \Delta t}$ leads to the accumulation of particles in regions of low diffusivity. In [5], L. Farnell and W. G Gibson have derived simple formulas for correcting the standard fixed-step MC method in the case of a spatially variable diffusion coefficient. We recall their MC algorithm in the next section.

\section{A biased random walk in a nonhomogeneous medium}

We suppose that the diffusion coefficient $D(x)>0$ varies with distance. Let $\Delta t$ be the time step and $L(x):=\sqrt{2 D(x) \Delta t}$ denote the uncorrected steplength. During the time 


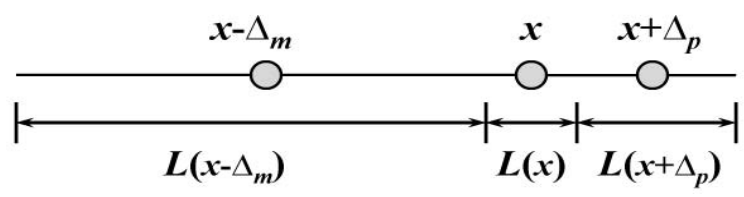

Figure 1. The lattice used for the random walk.

step, a particle at position $x$ moves to either $x-\Delta_{m}(x)$ or $x+\Delta_{p}(x)$. If we have

$$
\begin{aligned}
\Delta_{m}(x) & =\frac{1}{2}\left(L\left(x-\Delta_{m}(x)\right)+L(x)\right), \\
\Delta_{p}(x) & =\frac{1}{2}\left(L(x)+L\left(x+\Delta_{p}(x)\right)\right),
\end{aligned}
$$

the random walk stays on a (irregular) lattice: see Fig. 1.

Since $L(x)=\mathcal{O}(\sqrt{\Delta t})$, an approximate solution of (3.1)-(3.2) can be found from Taylor expansions:

$$
\begin{aligned}
& \Delta_{m}(x)=L(x)-\frac{1}{2} L(x) L^{\prime}(x)+\mathcal{O}\left((\Delta t)^{3 / 2}\right), \\
& \Delta_{p}(x)=L(x)+\frac{1}{2} L(x) L^{\prime}(x)+\mathcal{O}\left((\Delta t)^{3 / 2}\right) .
\end{aligned}
$$

Denote by $T_{\ell}(x)$ (resp. $T_{r}(x)$ ) the probability that a particle at $x$ moves to the left (resp. to the right) during $\Delta t$ : clearly $T_{\ell}(x)+T_{r}(x)=1$. If we consider a large number of particles, the average number $N(x, t)$ of particles at point $x$ and time $t$ satisfies:

$$
N(x, t+\Delta t)=N\left(x-\Delta_{m}, t\right) T_{r}\left(x-\Delta_{m}\right)+N\left(x+\Delta_{p}, t\right) T_{\ell}\left(x+\Delta_{p}\right),
$$

where $\Delta_{m}:=\Delta_{m}(x)$ and $\Delta_{p}:=\Delta_{p}(x)$. The concentration of particles at point $x$ and time $t$ is $c(x, t)=N(x, t) / L(x)$ and the time rate of change of concentration at point $x$ verifies:

$$
\frac{\partial c}{\partial t}(x, t)=\lim _{\Delta t \rightarrow 0} \frac{1}{L(x)} \frac{N(x, t+\Delta t)-N(x, t))}{\Delta t} .
$$

It follows then from (3.5):

$$
\begin{aligned}
\frac{\partial c}{\partial t}(x, t)= & \lim _{\Delta t \rightarrow 0} \frac{1}{L(x)} \frac{1}{\Delta t}\left(L\left(x+\Delta_{p}\right) c\left(x+\Delta_{p}, t\right) T_{\ell}\left(x+\Delta_{p}\right)\right. \\
& \left.+L\left(x-\Delta_{m}\right) c\left(x-\Delta_{m}, t\right) T_{r}\left(x-\Delta_{m}\right)-L(x) c(x, t)\right) .
\end{aligned}
$$

Using the Taylor expansions

$$
\begin{aligned}
c\left(x-\Delta_{m}, t\right) & =c(x, t)-\Delta_{m} \frac{\partial c}{\partial x}(x, t)+\frac{1}{2} \Delta_{m}^{2} \frac{\partial^{2} c}{\partial x^{2}}(x, t)+\mathcal{O}\left((\Delta)^{3 / 2}\right), \\
c\left(x+\Delta_{p}, t\right) & =c(x, t)+\Delta_{p} \frac{\partial c}{\partial x}(x, t)+\frac{1}{2} \Delta_{p}^{2} \frac{\partial^{2} c}{\partial x^{2}}(x, t)+\mathcal{O}\left((\Delta)^{3 / 2}\right)
\end{aligned}
$$


in Eq. (3.7), we get

$$
\frac{\partial c}{\partial t}(x, t)=\lim _{\Delta t \rightarrow 0} \frac{1}{\Delta t}\left(d_{0} c(x, t)+d_{1} \frac{\partial c}{\partial x}(x, t)+d_{2} \frac{\partial^{2} c}{\partial x^{2}}(x, t)\right),
$$

where

$$
\begin{aligned}
d_{0} & :=R_{p}(x) T_{\ell}\left(x+\Delta_{p}\right)+R_{m}(x) T_{r}\left(x-\Delta_{m}\right)-1 \\
d_{1} & :=R_{p}(x) \Delta_{p} T_{\ell}\left(x+\Delta_{p}\right)-R_{m}(x) \Delta_{m} T_{r}\left(x-\Delta_{m}\right), \\
d_{2} & :=\frac{1}{2}\left(R_{p}(x) \Delta_{p}^{2} T_{\ell}\left(x+\Delta_{p}\right)+R_{m}(x) \Delta_{m}^{2} T_{r}\left(x-\Delta_{m}\right)\right),
\end{aligned}
$$

together with $R_{m}(x):=L\left(x-\Delta_{m}\right) / L(x)$ and $R_{p}(x):=L\left(x+\Delta_{p}\right) / L(x)$. We recover the diffusion equation (2.1) if

$$
\lim _{\Delta t \rightarrow 0} \frac{d_{0}}{\Delta t}=0, \quad \lim _{\Delta t \rightarrow 0} \frac{d_{1}}{\Delta t}=D^{\prime}(x), \quad \lim _{\Delta t \rightarrow 0} \frac{d_{2}}{\Delta t}=D(x) .
$$

These conditions are satisfied if we have

$$
T_{\ell}(x)=\frac{1}{2}-\frac{1}{4} L^{\prime}(x)+\mathcal{O}(\Delta t) \quad \text { and } \quad T_{r}(x)=\frac{1}{2}+\frac{1}{4} L^{\prime}(x)+\mathcal{O}(\Delta t) .
$$

Details of the calculation are given in the original paper [5].

The corrected random walk can be summarized as follows: a particle at position $x$ at time $t$ should at time $t+\Delta t$, with probability $T_{\ell}(x)$ move to position $x-\Delta_{m}(x)$ and with probability $T_{r}(x)$ move to position $x+\Delta_{p}(x)$. Here

$$
\begin{array}{rlrl}
\Delta_{m}(x) & :=L(x)-\frac{1}{2} L(x) L^{\prime}(x), & T_{\ell}(x):=\frac{1}{2}-\frac{1}{4} L^{\prime}(x), \\
\Delta_{p}(x):=L(x)+\frac{1}{2} L(x) L^{\prime}(x), & T_{r}(x):=\frac{1}{2}+\frac{1}{4} L^{\prime}(x)
\end{array}
$$

and $L(x):=\sqrt{2 D(x) \Delta t}$ is the uncorrected steplength.

\section{A quasi-random walk on an asymmetrical lattice}

In this section, we develop a QMC approach for the simulation of the diffusion equation in a multi-dimensional nonhomogeneous medium. The algorithm is based on the QMC scheme described in Section 2 and uses the correction method of Section 3. We consider the pure initial value problem:

$$
\begin{aligned}
\frac{\partial c}{\partial t}(\mathbf{x}, t) & =\nabla \cdot(D \nabla c)(\mathbf{x}, t), \quad \mathbf{x} \in \mathbb{R}^{s}, t>0 \\
c(\mathbf{x}, 0) & =c_{0}(\mathbf{x}), \quad \mathbf{x} \in \mathbb{R}^{s},
\end{aligned}
$$


where the initial concentration $c_{0}(\mathbf{x}) \geq 0$ satisfies

$$
\int_{\mathbb{R}^{s}} c_{0}(\mathbf{x}) d \mathbf{x}=1 .
$$

This implies

$$
\forall t>0 \quad \int_{\mathbb{R}^{s}} c(\mathbf{x}, t) d \mathbf{x}=1 .
$$

Let $\Delta t$ be a time step and $t_{n}:=n \Delta t$, for $n \in \mathbb{N}$.

\subsection{Constant number of particles}

We first use a fixed number of particles. Let $b$ and $d_{1}, \ldots, d_{s}$ be integers. Put $m:=$ $d_{1}+\cdots+d_{s}$ and $N=b^{m}$. We are looking for an approximation of the solution at time $t_{n}$ of the form

$$
c^{n}:=\frac{1}{N} \sum_{0 \leq k<N} \delta\left(\mathbf{x}-\mathbf{x}_{k}^{n}\right),
$$

where $\mathbf{x}_{0}^{n}, \ldots, \mathbf{x}_{N-1}^{n}$ are the (positions of the) particles at time $t_{n}$. The QMC algorithm has several steps:

\subsubsection{Initialization}

The initial locations $\left\{\mathbf{x}_{0}^{0}, \ldots, \mathbf{x}_{N-1}^{0}\right\}$ are sampled from the probability distribution $c_{0}(\mathbf{x}) d \mathbf{x}$. This is done by mapping a $(0, m, s)$-net in base $b$ to $\mathbb{R}^{s}$. The choice of the mapping depends on the initial distribution to be sampled; in our numerical experiments (section 5), the particles are released from a point source at origin, so we take $\mathbf{x}_{0}^{0}=\cdots=\mathbf{x}_{N-1}^{0}:=\mathbf{0}$.

\subsubsection{Displacement of particles}

We use a low-discrepancy sequence $U=\left\{\mathbf{u}_{0}, \mathbf{u}_{1}, \ldots\right\} \subset I^{2 s}$ for moving the particles. For an integer $n$, denote $U^{n}:=\left\{\mathbf{u}_{j}: n N \leq j<(n+1) N\right\}$. Let $\Pi^{\prime}: I^{2 s} \rightarrow I^{s}$ be the projection defined by $\Pi^{\prime}\left(u_{1}, \ldots, u_{2 s}\right):=\left(u_{1}, \ldots, u_{s}\right)$; we assume that for all $n \in \mathbb{N}$,

$$
\Pi^{\prime} U^{n} \text { is a }(0, m, s) \text {-net in base } b \text {. }
$$

For $\mathbf{u} \in I^{2 s}$, we put $\mathbf{u}^{\prime}:=\Pi^{\prime}(\mathbf{u})$. We generalize formulas (3.13)-(3.14) as follows. If $L(\mathbf{x}):=\sqrt{2 D(\mathbf{x}) \Delta t}$, we define for $1 \leq i \leq s:$

$$
\begin{aligned}
\Delta_{m}^{i}(\mathbf{x}):=L(\mathbf{x})-\frac{1}{2} L(\mathbf{x}) \frac{\partial L}{\partial x_{i}}(\mathbf{x}), & T_{\ell}^{i}(\mathbf{x}):=\frac{1}{2}-\frac{1}{4} \frac{\partial L}{\partial x_{i}}(\mathbf{x}), \\
\Delta_{p}^{i}(\mathbf{x}):=L(\mathbf{x})+\frac{1}{2} L(\mathbf{x}) \frac{\partial L}{\partial x_{i}}(\mathbf{x}), & T_{r}^{i}(\mathbf{x}):=\frac{1}{2}+\frac{1}{4} \frac{\partial L}{\partial x_{i}}(\mathbf{x}) .
\end{aligned}
$$

Suppose that at time $t_{n}$, the particles are located at positions $\mathbf{x}_{0}^{n}, \ldots, \mathbf{x}_{N-1}^{n}$ : we determine the positions at time $t_{n+1}$ in two steps. 


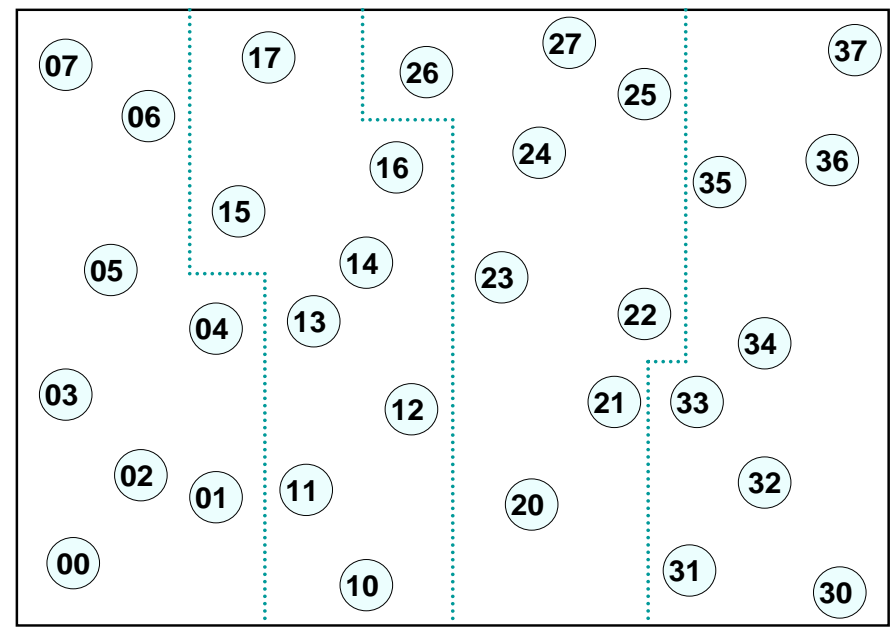

Figure 2. Sorting $2^{2+3}=32$ particles $\left(b=2, s=2, d_{1}=2, d_{2}=3\right)$

Relabeling the particles. The particles are labeled $\mathbf{x}_{\mathbf{a}}^{(n)}$ using a multi-dimensional index $\mathbf{a}=\left(a_{1}, \ldots, a_{s}\right)$ with $0 \leq a_{i}<b^{d_{i}}$ for $1 \leq i \leq s$, such that if $\mathbf{a}$ and $\widetilde{\mathbf{a}}$ are distinct indices, then

$$
a_{1}=\widetilde{a}_{1}, \ldots, a_{i-1}=\widetilde{a}_{i-1}, a_{i}<\widetilde{a}_{i} \Rightarrow x_{\mathbf{a}, i}^{n} \leq x_{\widetilde{\mathbf{a}}, i}^{n} .
$$

This type of sorting was first used in [13] and generalizes the classical one-dimensional sorting of (2.14). This is done in $s$ steps as follows: the values of the first coordinate $x_{1}$ of the particles are used to sort them into $b^{d_{1}}$ subsets of level 1 . In each subset of level 1 , the values of the second coordinate $x_{2}$ of the particles are used to sort them into $b^{d_{2}}$ subsets of level 2, and so on: see Fig. 2 for an example.

Performing a random walk We use the quasi-random points $\mathbf{u}_{j}$. To each $\mathbf{u}^{\prime}=$ $\left(u_{1}, \ldots, u_{s}\right) \in I^{s}$, we associate the index $\mathbf{a}\left(\mathbf{u}^{\prime}\right):=\left(\left\lfloor b^{d_{1}} u_{1}\right\rfloor, \ldots,\left\lfloor b^{d_{s}} u_{s}\right\rfloor\right)$. It follows from (4.5) that the mapping

$$
j \in\{n N, n N+1, \ldots,(n+1) N-1\} \mapsto \mathbf{a}\left(\mathbf{u}_{j}^{\prime}\right)
$$

is a bijection onto $\left\{0, \ldots, b^{d_{1}}-1\right\} \times \cdots \times\left\{0, \ldots, b^{d_{s}}-1\right\}$. At time $t_{n+1}$, the coordinates of the particles are updated as follows: for $n N \leq j<(n+1) N$ and $1 \leq i \leq s$,

$$
x_{\mathbf{a}\left(\mathbf{u}_{j}^{\prime}\right), i}^{n+1}= \begin{cases}x_{\mathbf{a}\left(\mathbf{u}_{j}^{\prime}\right), i}^{n}-\Delta_{m}^{i}\left(\mathbf{x}_{\mathbf{a}\left(\mathbf{u}_{j}^{\prime}\right)}^{n}\right), & \text { if } u_{j, s+i}<T_{\ell}^{i}\left(\mathbf{x}_{\mathbf{a}\left(\mathbf{u}_{j}^{\prime}\right)}^{n}\right), \\ x_{\mathbf{a}\left(\mathbf{u}_{j}^{\prime}\right), i}^{n}+\Delta_{p}^{i}\left(\mathbf{x}_{\mathbf{a}\left(\mathbf{u}_{j}^{\prime}\right)}^{n}\right), & \text { otherwise. }\end{cases}
$$


The first $s$ coordinates of the quasi-random point $\mathbf{u}_{j}$ are used to select the particle, while the last $s$ coordinates are used to update the coordinates of the particle.

\subsection{Varying number of particles}

The QMC algorithm of [2] works with a constant number $N$ of numerical particles and $N$ is equal to a power of some prime base $b$. This is required if we want the mapping (2.19) to be a bijection. In an application of interest to us, physical particles are released from a source so that the number of particles increases over time. We can overcome the difficulty as follows. If $N$ is the number of particles at time $t_{n}$, we write the digit expansion of $N$ in base $b$ :

$$
N=\sum_{h=0}^{\infty} \alpha_{h}(N) b^{h},
$$

where $0 \leq \alpha_{h}(N)<b$ and $\alpha_{h}(N)=0$ for sufficiently large $h$. The set of $N$ particles is split into $\alpha_{0}(N)$ subsets of size $b^{0}, \alpha_{1}(N)$ subsets of size $b^{1}$, and so forth. Each subset is treated separately (for relabeling and displacement). At the end of the time step all the subsets are merged in one set of $N$ particles before adding new particles that are released from the source during the time step.

\section{Numerical experiments}

In this section, we present the results of numerical experiments which illustrate the precision of the QMC scheme. We focus on biological applications with $\mathrm{Ca}^{2+}$ diffusion inside the neuromuscular junction of the crayfish [15]. Calcium ions are released from a point source as the result of a train of action potentials arriving at that location, with each impulse resulting in release. The ions then diffuse independently through the surrounding medium and act on receptors at varying distances from the release point, chosen as the origin of the axes. The diffusion coefficient varies with position. The number of particles released from the source is $N=2^{14}$. We have considered the following cases in dimensions 1 and 2:

(i) instantaneous emission of particles at $t=0$;

(ii) emission of particles during a time interval $\left[0, \tau_{e}\right]$.

We evaluate the concentration of particles in a region of space as the number of particles in the region divided by the total number of particles. We compute the evolution of concentration in different regions during a time interval $\left[0, T_{f}\right]$. The time step is $\Delta t=1.610^{-4}$. In both cases $\tau_{e}:=1.31072=2^{13} \Delta t$ and $T_{f}:=10=62500 \Delta t$. We compare the results given by the QMC scheme with those produced by the MC scheme of [5]. Since no analytical solution is available, a reference solution is obtained by using a MC simulation with a very large number of simulation particles. 


\subsection{One-dimensional diffusion}

We consider the spatially varying diffusion coefficient used in [4, 5]:

$$
D(x):=\widehat{D}(1-0.8 u(x)),
$$

where $\widehat{D}:=4000$ and

$$
u(x):=\frac{1}{2}(\tanh (A(b-x))+1), \quad A=3.510^{-2}, \quad b=200 .
$$

The quasi-random sequence used in 1-D simulations is the $(0,2)$-sequence of Faure in base $b=2$ (see [17]). We compare MC and QMC results by calculating the time evolution of the concentration of ions in the space intervals $[20,40]$ and $[60,80]$. To smooth the curves and to make comparison clearer, we average the results over a time interval of amplitude 0.16 . The reference solution is obtained by a MC simulation using a sample $2^{6}$ times larger than the original one, i.e., with $2^{20}$ particles.

\subsubsection{Instantaneous emission}

All the particles are released at time $t=0$ from the point source. The concentrations are displayed in Fig. 3. Using quasi-random numbers in place of pseudo-random numbers and reordering the particles clearly reduce scattering of the results.

\subsubsection{Non-instantaneous emission}

Now the ions are released over the time interval $\left[0, \tau_{e}\right]$ : two particles are emitted at each time step $\Delta t$. Figure 4 shows the outputs of the computations. Once again, the quasi-random strategy produces more accurate approximations than the standard random walk method, and the splitting technique used during emission seems to be efficient.

\subsection{Two-dimensional diffusion}

The diffusion coefficient is that of Eq. (5.1), with $x$ replaced by the radial coordinate $r:=\sqrt{x_{1}^{2}+x_{2}^{2}}$. Here, the low-discrepancy sequence used is the $(3,4)$-sequence of Niederreiter in base $b=2$ (see [17]). We compare MC and QMC results by calculating the time evolution of the concentration of ions in the squares $[20,40] \times[10,30]$ and $[60,80] \times[10,30]$. We average the results over a time interval of amplitude 0.24 . The reference solution is obtained by a MC simulation using a sample $2^{7}$ times larger than the original one, i.e., with $2^{21}$ particles.

\subsubsection{Instantaneous emission}

At $t=0$, all the particles are released from the point source. The concentrations are displayed in Fig. 5. The improvement due to QMC is clear even in dimension 2. 

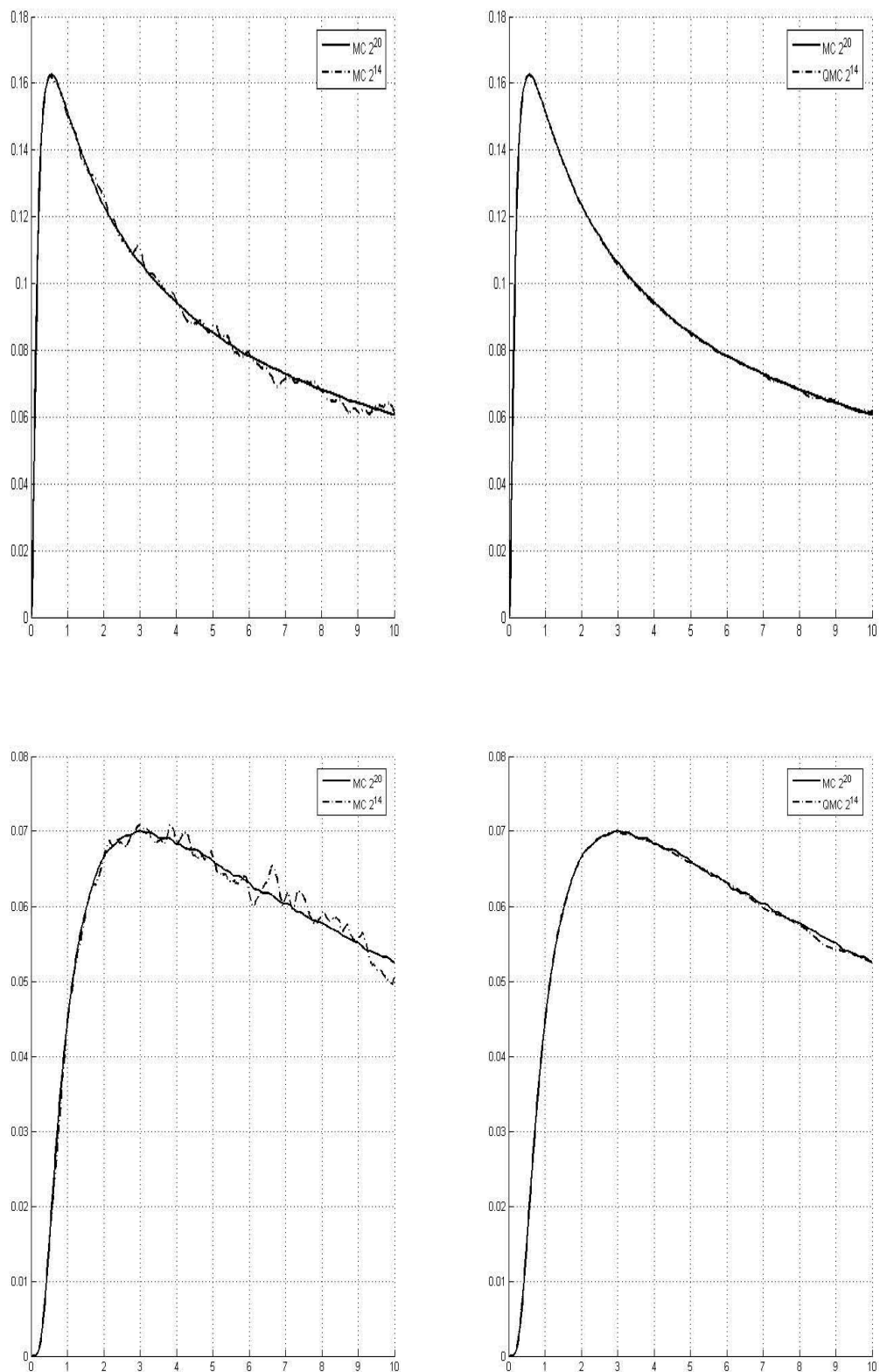

Figure 3. Instantaneous emission in one-dimensional medium. Time evolution of the concentration in the intervals $[20,40]$ (up) and [60, 80] (down). Comparison of MC (left) and QMC (right) simulations with a reference solution. 

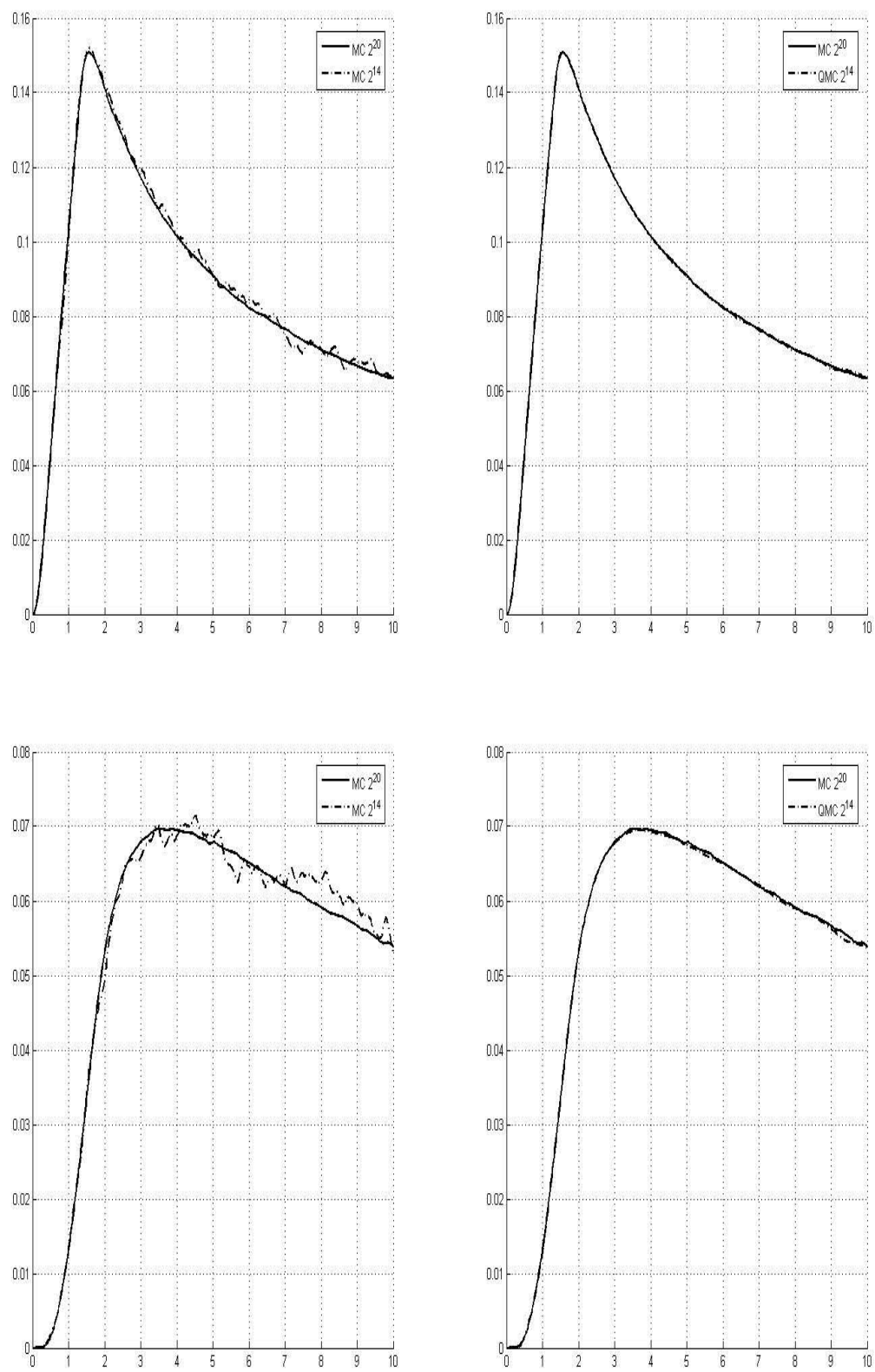

Figure 4. Non-instantaneous emission in one-dimensional medium. Time evolution of the concentration in the intervals $[20,40]$ (up) and $[60,80]$ (down). Comparison of MC (left) and QMC (right) simulations with a reference solution. 

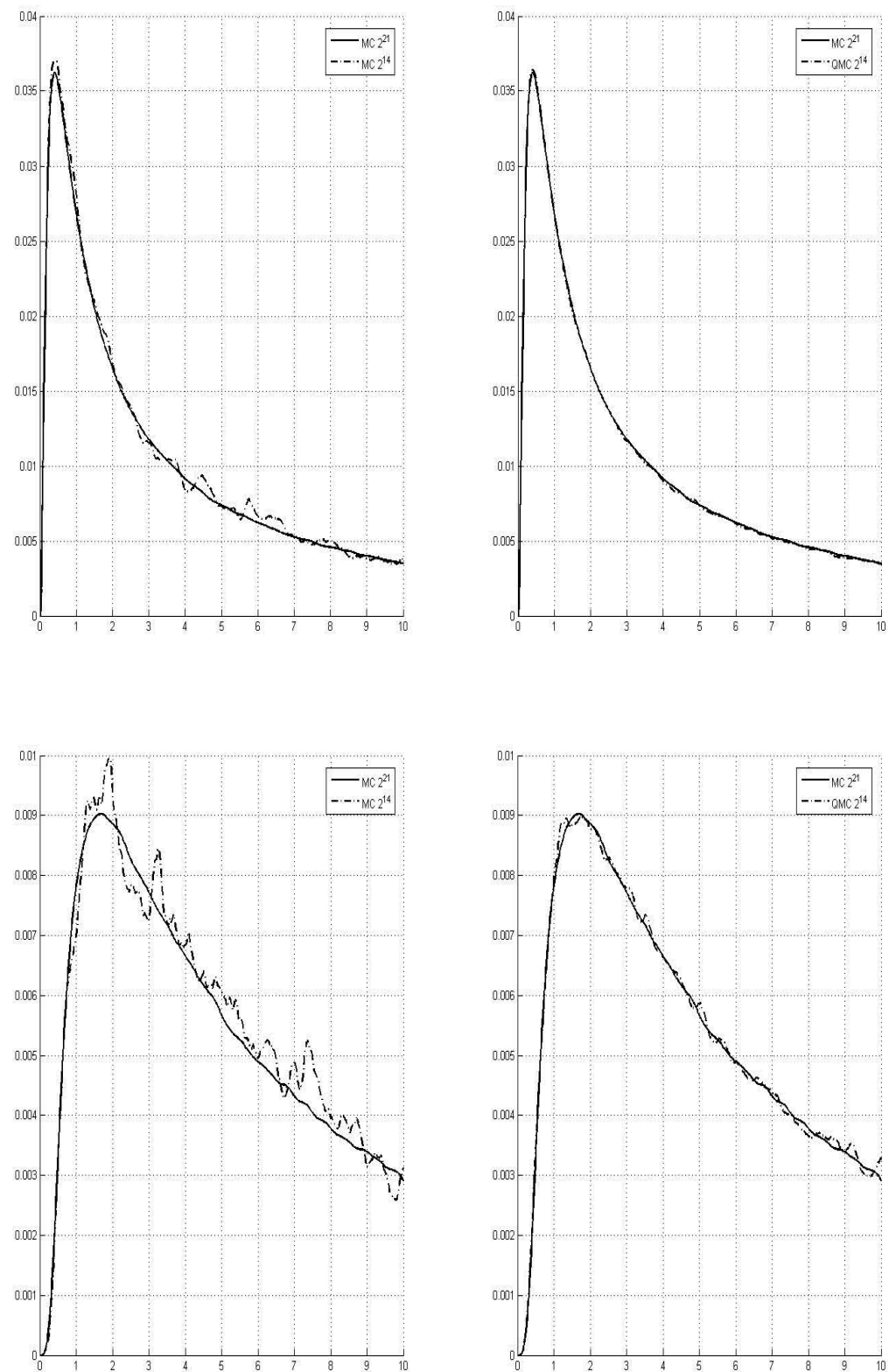

Figure 5. Instantaneous emission in two-dimensional medium. Time evolution of the concentration in the squares $[20,40] \times[10,30]$ (up) and $[60,80] \times[10,30]$ (down). Comparison of MC (left) and QMC (right) simulations with a reference solution. 


\subsubsection{Non-instantaneous emission}

During $\tau_{e}$, two particles are emitted from the source at every time step $\Delta t$. The results of MC and QMC simulations, as plotted on Fig. 6, show the superiority of QMC approach.

\section{Conclusion}

We have presented a QMC algorithm for the simulation of diffusion processes in spatially nonhomogeneous media. The scheme is a generalization of the QMC method described in [2] in the case of a constant diffusion coefficient. The diffusing substance is approximated by a set of $N$ particles, which are sampled at $t=0$ from the initial distribution. Time is discretized, and the particles are reordered according to their coordinates at the beginning of every time step. A mapping between a low-discrepancy sequence and the set of the positions of the particles determines the movement of each of them. The steps of the random walk are corrected according to formulas derived in [5]. The number $N$ is allowed to change during the simulation. The results of some numerical examples have shown that the new QMC method is more accurate than the standard MC scheme. Directions for future work include the study of theoretical convergence of the method and its application to more general diffusion problems with boundary conditions.

\section{References}

1. A. J. Chorin, Numerical study of slightly viscous flow, J. Fluid Mech. 57 (1973), pp.785796.

2. I. Coulibaly and C. Lécot, Simulation of diffusion using quasi-random walk methods, Math. Comput. Simul. 47 (1998), pp. 153-163.

3. R. El Haddad, C. Lécot and G. Venkiteswaran, Quasi-Monte Carlo simulation of diffusion in a spatially nonhomogeneous medium, in: Monte Carlo and Quasi-Monte Carlo Methods 2008, pp. 339-354, Springer, Berlin, 2010.

4. L. Farnell and W. G. Gibson, Monte Carlo simulation of diffusion in a spatially nonhomogeneous medium: correction to the Gaussian steplength, J. Comput. Phys. 198 (2004), pp. $65-79$.

5. L. Farnell and W. G. Gibson, Monte Carlo simulation of diffusion in a spatially nonhomogeneous medium: A biased random walk on an asymmetrical lattice, J. Comput. Phys. 208 (2005), pp. 253-265.

6. W. Feller, An Introduction to Probability Theory and its Applications, vol. 1, 3rd ed., John Wiley \& Sons, New York, 1968.

7. A. L. Fogelson and R. H. Dillon, Optimal smoothing in function-transport particle methods for diffusion problems, J. Comput. Phys. 109 (1993), pp. 155-163.

8. A. F. Ghoniem and F. S. Sherman, Grid-free simulation of diffusion using random walk methods, J. Comput. Phys. 61 (1985), pp.1-37. 

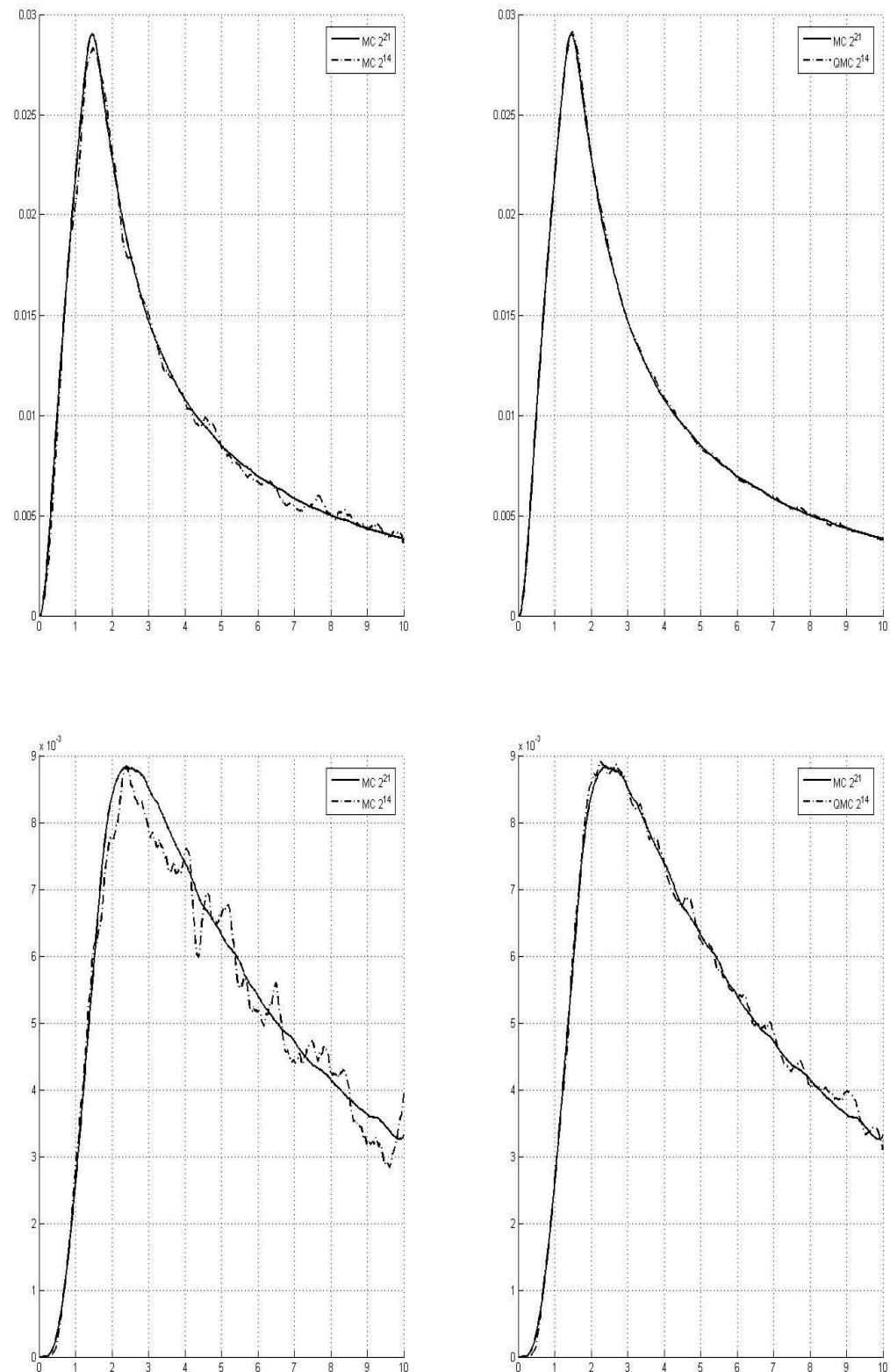

Figure 6. Non-instantaneous emission in two-dimensional medium. Time evolution of the concentration in the squares $[20,40] \times[10,30]$ (up) and $[60,80] \times[10,30]$ (down). Comparison of MC (left) and QMC (right) simulations with a reference solution. 
9. O. H. Hald, Convergence of random methods for a reaction-diffusion equation, SIAM J. Sci. Stat. Comput. 2 (1981), pp. 85-94.

10. J. R. Hunter, P. D. Craig and H. E. Phillips, On the use of random walk models with spatially variable diffusivity, J. Comput. Phys. 106 (1993), pp. 366-376.

11. M. Kac, Random walk and the theory of Brownian motion, Am. Math. Mon. 54 (1947), pp. 369-391.

12. C. Lécot, A direct simulation Monte Carlo scheme and uniformly distributed sequences for solving the Boltzmann equation, Computing 41 (1989), pp. 41-57.

13. C. Lécot and I. Coulibaly, A quasi-Monte Carlo scheme using nets for a linear Boltzmann equation, SIAM J. Numer. Anal. 35 (1998), pp. 51-70.

14. C. Lécot and F. El Khettabi, Quasi-Monte Carlo simulation of diffusion, J. Complexity 15 (1999), pp. 342-359.

15. V. Matveev, A. Sherman and R. S. Zucker, New and corrected simulations of synaptic facilitation, Biophys. J. 83 (2002), pp. 1368-1373.

16. W. J. Morokoff and R. E. Caflisch, A quasi-Monte Carlo approach to particle simulation of the heat equation, SIAM J. Numer. Anal. 30 (1993), pp. 1558-1573.

17. H. Niederreiter, Random Number Generation and Quasi-Monte Carlo Methods, SIAM CBMS-NSF Regional Conference Series in Applied Mathematics 63, SIAM, Philadelphia, 1992.

18. G. Venkiteswaran and M. Junk, Quasi-Monte Carlo algorithms for diffusion equations in high dimensions, Math. Comput. Simul. 68 (2005), pp. 23-41.

\section{Received In}

\section{Author information}

Rami El Haddad, Département de Mathématiques, Faculté des Sciences, Université SaintJoseph, BP 11-514 Riad El Solh, Beyrouth 1107 2050, Liban.

Email: rami.haddadefs.usj.edu. lb

Christian Lécot, Laboratoire de Mathématiques, UMR 5127 CNRS \& Université de Savoie, Campus scientifique, 73376 Le Bourget-du-Lac Cedex, France.

Email: Christian.Lecoteuniv-savoie.fr

Gopalakrishnan Venkiteswaran, Department of Mathematics, Birla Institute of Technology and Science, Vidya Vihar Campus, Pilani, 333031 Rajasthan, India.

Email: gvenki@bits-pilani.ac.in 